Notfall Rettungsmed 2010 · 13:347-348

DOI 10.1007/s10049-009-1270-6

Online publiziert: 18. Juli 2010

(c) Springer-Verlag 2010

\author{
C. K. Lackner $\cdot$ K. Burghofer \\ Institut für Notfallmedizin und Medizinmanagement \\ (INM), Klinikum der Universität München,
}

\title{
Dream Teams are made - not born!
}

Die weit überwiegende Mehrzahl von risikoreichen und gleichzeitig prognoserelevanten Handlungen am akutmedizinischen Patienten erfolgt nicht durch Einzelne, sondern ist die Resultante einer (ad hoc-)Teamleistung.

Eine insbesondere bei Führungspersonen immer noch weit verbreitete Meinung in diesem Zusammenhang besteht darin, zu unterstellen, dass eine Teamleistung dann erfolgreicher und nachhaltiger in Aspekten der Patientensicherheit würde, wenn alle Beteiligten „sich nur mal richtig anstrengen würden, um ein gutes Team zu sein“.

Intrinsische Motivation und (sehr) positive Haltung der Handelnden gegenüber der Aufgabenstellung sind insbesondere in der Akutmedizin außergewöhnlich gut ausgeprägt. Ein Umstand, um den uns viele sog. Risikobranchen beneiden. Eine Ressource, die wertvoll und von zentraler Bedeutung ist.

Die hohe Motivation und positive Haltung existieren, trotz der bisweilen widrigen institutionellen Rahmenbedingungen (z. B. hohe Arbeitsbelastung, wenig positive Rückmeldungen, steile Hierarchien) und einer noch immer in einigen Einrichtungen inhärenten sogenannten „culture of blame“ (name -> blame -> shame) statt einer gemeinsamen Sicherheitskultur.

Akzeptiert man die stabilen wissenschaftlichen Befunde der vergangenen Jahre, dass die Ursache von relevanten Fehlerketten, die letztlich zu Zwischenfällen in der Akutmedizin führen, in über
70\% den „Human Factors“ zuzurechnen sind und somit potenziell vermeidbar wären, gewinnt Team Resource Management eine zentrale Bedeutung.

\section{- Ein professionelles Team braucht ein gemeinsames Hauptziel, mit dem kollektives Einverständnis besteht und das allen Mitgliedern erstrebenswert erscheint.}

Neben dem Wohl des Patienten ist die Patientensicherheit in Risikoumgebungen wie der Akutmedizin ein häufig benanntes Hauptziel dieser Teams.

Daneben gibt es individuelle Teilziele, die zu erreichen das Team und jedes Mitglied ein besonderes Interesse haben. Die Wirksamkeit eines professionellen Teams hängt ganz wesentlich vom Einverständnis der Team-Mitglieder über das Hauptziel und von ihrer Bereitschaft ab, ihre persönlichen Ziele in den Dienst des Hauptziels zu stellen.

Das entscheidende Erfolgskriterium eines Teams in der Akutmedizin ist seine Leistungsfähigkeit und die gemeinsame Zielorientierung. Erfolgreiche Teams sind in der Lage, Leistungen und Ergebnisse für Patienten zu erreichen, welche durch einzelne Mitglieder niemals erreicht werden könnten. Ihre individuellen Stärken addieren sich im Team und kompensieren individuelle Schwächen. Die kollektive Qualität der Zielerreichung ist weit mehr als die Summe der Einzelbegabungen.

Das Leitthema dieser Ausgabe von Notfall + Rettungsmedizin widmet sich in
4 Übersichtarbeiten dem Thema Team Resource Management in der Akutmedizin.

Die Entscheidung hierzu entstand aus der klaren wissenschaftlichen Erkenntnislage, dass Patientensicherheit auf erfolgreicher interdisziplinärer, interprofessioneller Teamarbeit basiert.

M. Rall et al. [4] geben in der Übersicht „Crisis Resource Management (CRM) - der Faktor Mensch in der Akutmedizin“ einen Überblick über wesentliche Erkenntnisse der Human Factor-Forschung und stellen etablierte Konzepte im Bereich des CRM-Trainings vor. Sie zeigen bewährte Ausbildungs- und Trainingsformate für den Human Factor-Themenkontext, insbesondere CRM-basiertes Simulations-Team-Training mit videogestützten Nachbesprechungen auf, die sich als besonders effektiv und nachhaltig erwiesen haben. Daneben findet sich ein Ausblick auf weitere erfolgreiche Konzepte, welche eine Erhöhung der Sicherheitskultur bewirken und wie die Einführung nichtpunitiver Incident-Reporting-Systeme als wichtiger Bestandteil für die nachhaltige Erhöhung der Patientensicherheit in der Zukunft zu werten ist.

Im wissenschaftlichen Sektor, der sich mit Fragestellungen zur Patientensicherheit beschäftigt, wird die Rolle der effektiven Teamarbeit gegenwärtig intensiv und sehr breit diskutiert. Besonders intensive Diskussionen induzierte die sich immer wieder bestätigende Erkenntnislage, dass kritische Ereignisse in der Akutmedizin nicht auf einen Mangel an klinischer Kompetenz reduziert werden können, 
sondern in der überwiegenden Mehrzahl maßgeblich auf inadäquate Zusammenarbeit im Team zurückzuführen sind.

T. Manser [3] gibt mit „Koordination und Teamwork in der Akutmedizin“ einen Überblick über zentrale Konzepte der Teamforschung in Hochrisiko-Industrien und über aktuelle Forschungsarbeiten zur adaptiven Koordination in der Akutmedizin. Solche wissenschaftliche Erkenntnisse können wesentlich zu einer Erhöhung der Patientensicherheit beitragen, wenn sich aus dem Wissenstransfer aus anderen Hochrisiko-Industrien für die Akutmedizin spezifische Empfehlungen für Trainingsmaßnahmen und Arbeitssystemgestaltung ableiten lassen.

Eine ganz zentrale Rolle für erfolgreiche Teamarbeit nimmt die Kommunikation im Team ein.

K. Burghofer et al. [1] machen in ihrer Übersichtsarbeit „Kommunikation - Risikofaktor in der Akutmedizin“ deutlich, dass Patientensicherheit in der Akutmedizin nur mit professioneller Kommunikation möglich ist. Lange Zeit wurde der Kommunikation in der medizinischen Aus-, Fort- und Weiterbildung wenig oder keine Beachtung zuteil. Erst mit zunehmenden wissenschaftlichen Erkenntnissen im Sektor der Patientensicherheit und der Aufarbeitung von Zwischenfällen und Fehlern in der Medizin wurde deutlich, dass diesen häufig ein Kommunikationsdefizit zugrunde liegt.

Die Akutmedizin mit ihrem hohen Komplexitätsgrad, der Vielzahl von Schnittstellen, ihren beteiligten Akteuren aus unterschiedlichen Fachrichtungen und Professionalisierungshintergründen sowie dem immanentemZeitdruckstellt eine Hochrisikoumgebung dar. Vor dem Hintergrund von Kommunikationstheorien und-modellen werden von Burghofer Ansätze aufgezeigt, wie kommunikative Prozesse zielführender gestaltet werden können, so dass aus dem Risikofaktor Kommunikation eine Qualitätsressource für akutmedizinische Patientensicherheit werden kann.

B. Lang et al. [2] beschreiben in ihrer Übersicht „Team Resource Management in der Luftrettung “ den sehr erfolgreichen Weg, ein unternehmensübergreifendes CRM-Konzept für die deutschsprachige Luftrettung konsent zu definieren und nachhaltig gemeinsam zu implementie- ren. Das Format und das damit korrespondierende ACRM-Train-the-TrainerKonzept kann als Rollenmodell für weitere Handlungsfelder in der Akutmedizin bestens Anwendung finden. Dies stellt ein hervorragendes Bespiel gelebter Sicherheitskultur von Luftrettungsunternehmen dar.

Erfolgreiche professionelle Teams haben vor der gemeinsamen Aufgabenbewältigung Fragen zu Führungsverhalten, Arbeitsstil, Organisation und die Rollenverteilung klar geregelt. Derartige TeamStrukturen sind auf die zu lösende Aufgabe zielorientiert abgestimmt; individuelle Fähigkeiten und Teilaufgaben werden strategisch sinnvoll koordiniert. Teammitglieder haben trainiert, (informelle) Führungsansprüche zurückzustellen, Rücksicht aufeinander zu nehmen und Konkurrenzdenken und Emotionen aus dem Kontext der unmittelbaren Aufgabenbewältigung zu verbannen.

Erfolgreiche Teams sind in der Lage, flexibel, aufmerksam, methodisch und zielbewusst zu interagieren.

All dies ist im Team Ressource Management erfolgreich und nachhaltig für jeden Teilnehmer in der Gruppe trainierbar. Es gibt in der Medizin wenige vergleichbare Trainingsformate, die dies ebenfalls für sich beanspruchen können.

Ein Team ist somit eine aktive professionelle Gruppe von Menschen, die sich auf gemeinsame Ziele verpflichtet haben, interdisziplinär und interprofessionell.

Mit Freude an der gemeinsamen Teamarbeit erreichen sie hervorragende Leistungen. Diese Konstellation entsteht nicht aus sich - sie muss erarbeitet und kann erfolgreich trainiert werden.

Dream Teams are made - not born!

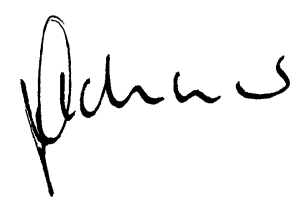

C. K. Lackner

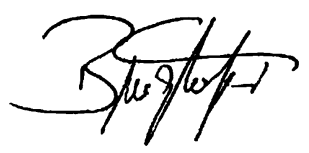

K. Burghofer

\section{Korrespondenzadresse \\ Prof. Dr. C. K. Lackner}

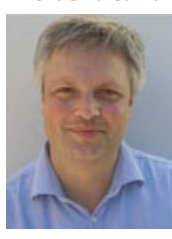

Institut für Notfallmedizin und Medizinmanagement (INM), Klinikum der Universität München

Schillerstr. 53, 80336 München christian.lackner@med. uni-muenchen.de

\section{Literatur}

1. Burghofer K, Lackner CK (2010) Kommunikation Risikofaktor in der Akutmedizin. Notfall Rettungsmed 5: doi 10/1007/s10049-009-1273-3

2. Lang B, Ruppert M, Schneibel W, Urban B (2010) Teamtraining in der Luftrettung - Aeromedical Crew Resource Management. Ein europäisches Trainingsprogramm zur Optimierung der Flugund Patientensicherheit in der Luftrettung. Notfall Rettungsmed: doi 10/1007/s10049-009-1274-2

3. Manser T (2010) Koordination und Teamwork in der Akutmedizin. Notfall Rettungsmed 5: doi 10/1007/s10049-009-1272-4

4. Rall M, Lackner CK (2010) Crisis Resource Management (CRM) - der Faktor Mensch in der Akutmedizin. Notfall Rettungsmed 5: doi 10/1007/s10049009-1271-5 